\title{
Regarding: "Localized Marked Elongation of the Distal Internal Carotid Artery with or without PHACE Syndrome: Segmental Dolichoectasia of the Distal Internal Carotid Artery"
}

W e read with interest the recently published article by Jia et al. ${ }^{1}$ The authors reported a consecutive case series of 20 patients with striking arterial elongations with marked tortuosity and/or a conglomerated vascular mass involving the distal ICA. The authors reported that these lesions were associated with a striking female predominance (9:1, F/M) and a relatively young median age of 43.5 years. In general, the vascular lesions were incidental findings, and very few patients had any medical comorbidities. The authors reported a benign natural history for these lesions with slow progression of the lesions on follow-up imaging. Most interestingly, 9 of 20 patients had mild atrophy of the ipsilateral midbrain, and 4 of 20 patients met all the diagnostic criteria for PHACE syndrome. ${ }^{1}$

The arterial lesions in the series of Jia et $\mathrm{al}^{1}$ have been previously described as "pure arterial malformations," defined as dilated, overlapping, and tortuous arteries forming a mass of arterial loops with a coil-like appearance in the absence of any arteriovenous connection. ${ }^{2}$ In a recently published case series, our group identified 12 cases of pure arterial malformations. ${ }^{3}$ Similar to Jia et al, we found a striking female predominance $(83.3 \%)$ and found that these patients were relatively young (mean age, 26.2 years). The lesions were incidental in all cases. Pure arterial malformations affected the anterior cerebral arteries in 25\% of cases, the posterior communicating artery (PcomA) segment in 33.3\% of cases, the MCA in $16.6 \%$ of cases, and posterior circulation arteries in $25 \%$ of cases. Four patients had focal aneurysms, and 5 lesions were partially calcified. Like Jia et al, we found that lesions affecting the PcomA segment of the ICA went on to affect the PcomA and posterior cerebral artery (PCA). Many of the lesions reported in our article had a striking resemblance to those reported by Jia et al. After 2.5 years of follow-up, none of the lesions had associated ischemia or hemorrhage, suggesting a benign natural history. ${ }^{3}$

We agree with the general assessment of Jia et $\mathrm{al}^{1}$ that these lesions have a benign natural history and do not warrant treatment. However, imaging follow-up every few years may be beneficial so that we can better understand the natural history of these lesions. Our group suspects that these lesions likely form early in life or in utero. ${ }^{4}$ Potential etiologies that have been discussed in the literature include a congenital defect or insult resulting in arterial dysplasia; an insult such as a viral infection or somatic mutation occurring later in life affecting a particularly vulnerable arterial segment (ie, segmental arterial vulnerability); or chronic healed dissection.

There have been at least 20 reported cases of pure arterial malformations reported in the literature before $2017 .^{3}$ In general, these patients are younger, and most patients are female. More than $80 \%$ of cases are discovered incidentally. Lesions involving the distal anterior cerebral arteries (ACAs) are characterized as ectatic and moderately tortuous and have a looser coil configuration. Furthermore, calcifications of distal ACA lesions are relatively common in previously reported cases. These findings are like those we have seen with lesions involving the ACAs. Meanwhile, lesions of the MCA or PcomA-PCA are often composed of a tightly wound coil of vessels with superimposed clusters of aneurysms and calcifications. Previously reported lesions of the superior cerebellar artery and posterior inferior cerebellar artery also bear a remarkably similar resemblance to our cases in that the vessels have the appearance of a tightly wound coil without substantial ectasia or any associated aneurysm.

We are glad to see additional cases of pure arterial malformations reported in the literature. We encourage other authors to report their cases as they encounter them so the neurovascular community can be better informed regarding the appearance and natural history of these lesions.

\section{REFERENCES}

1. Jia ZY, Zhao LB, Lee DH. Localized marked elongation of the distal internal carotid artery with or without PHACE syndrome: segmental dolichoectasia of the distal internal carotid artery. AJNR Am J Neuroradiol 2018;39:817-23 CrossRef Medline

2. McLaughlin N, Raychev R, Duckwiler G, et al. Pure arterial malformation of the posterior cerebral artery: importance of its recognition. J Neurosurg 2013;119:655-60 CrossRef Medline

3. Brinjikji W, Cloft HJ, Flemming KD, et al. Pure arterial malformations. J Neurosurg 2017 Sep 29. [Epub ahead of print] CrossRef Medline

4. Sorenson TJ, Brinjikji W, Flemming KD, et al. Pure arterial malformation (PAM) of the posterior inferior cerebellar artery (PICA) with interspersed adipose tissue: a case report. J Neurosurg Ped. In press

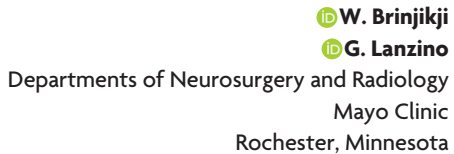

(D)W. Brinjikj Radiology Rochester, Minnesota 